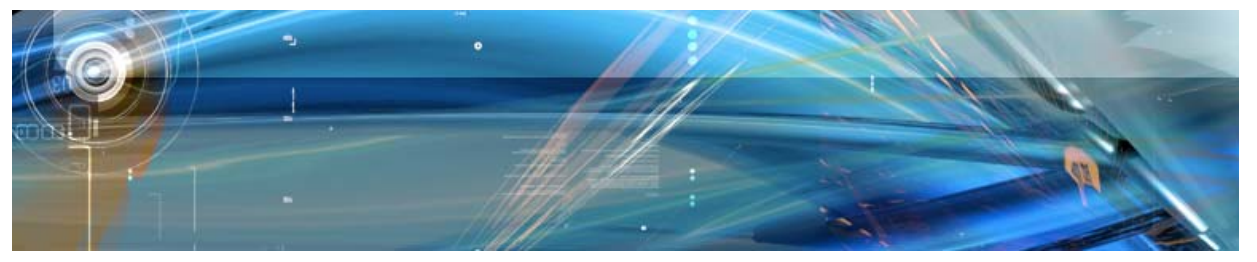

\title{
El estudio de los estereotipos a través del análisis de relatos ${ }^{1}$
}

\section{The Study of Stereotypes Through the Analysis of Narrations}

Francisco Bernete García ${ }^{(*)}$

Universidad Complutense de Madrid - España

\begin{abstract}
Resumen
El trabajo reseña una investigación donde se abordó el análisis de unas narraciones (libros de texto utilizados en Chile, Cuba, México, Perú y España, para explicar la asignatura de "Historia”) en su condición de relatos generados en sociedades con diferentes dinámicas históricas, culturales, políticas, etc. $\Upsilon$, consecuentemente, se concluye proponiendo el sentido que cabe atribuir a los resultados teniendo en cuenta el lugar donde se han producido y usado tales narraciones. Con esta investigación se pretendía comprender algo mejor los endoestereotipos y exoestereotipos nacionales, relacionando su construcción comunicativa con motivaciones y necesidades que no estaban explícitas en los textos
\end{abstract}

\begin{abstract}
This work summarizes an investigation on the analysis of narrations (textbooks used in Chile, Cuba, México, Peru and Spain, to explain the "History" subject) as stories generated in societies with different historical, cultural and political dynamics. Subsequently, it concludes proposing the meaning that must be attributed to the results, taking into consideration the place where such narrations have been produced and used. This investigation pursues a better understanding of the national endostereotypes and exo-stereotypes, relating their communicative construction with motivations and needs. Albeit such needs were not explicit in the History textbooks, they can be brought to light recur-
\end{abstract}

\footnotetext{
1 Texto publicado originalmente en la Revista Española de Investigaciones Sociológicas, $\mathrm{n}^{\circ}$ 57, enero-marzo de 1992, Madrid, C.I.S., pp. 123-135.
}

Mediaciones Sociales, № 3, II semestre de 2008, pp. 73-90. ISSN electrónico: 1989-0494. 
«El estudio de los estereotipos a través del análisis de relatos»

de historia, pero que podían ser traídos a la luz, recurriendo al análisis de contenido, cuando éste se lleva a cabo con técnicas adecuadas para estudiar las funciones mediadoras de las narraciones; entendiendo por mediación una "actividad que impone limites a lo que puede ser dicho y a las maneras de decirlo, por medio de un sistema de orden" (Martín Serrano, 1977: 54).

Palabras clave: mediación, discurso, estereotipo, análisis de contenido, libros de texto de historia, Latinoamérica. ring to content analysis, when it is conducted with the appropriate techniques to study the mediating functions of narrations. By mediation we mean an "activity that sets limits to what can be said and the ways of saying it, by means of a system of order" (Martin Serrano, 1977: 54).

Keywords: mediation, discourse, stereotype, content analysis, History textbooks, Latin America. 


\section{INTRODUCCIÓN}

El objeto del presente artículo es mostrar que el análisis de contenido de productos comunicativos ${ }^{2}$ puede usarse como una vía de aproximación a problemas tradicionalmente abordados por las Ciencias Sociales, desde diversas perspectivas. Entre ellos, los relativos a la estereotipia, el prejuicio o el etnocentrismo. Y, en general, los que se refieren a las "identidades colectivas".

Frederic Munné ha señalado cuáles son las principales aproximaciones teóricas al estudio de la formación de los estereotipos (Munné i Matamala, 1989). Para este autor, las explicaciones más polarizadas sobre cómo se forman y se desarrollan los estereotipos, son las de tipo psicoanalítico, donde se pone el acento en las necesidades insconscientes que satisfariamos al construir estereotipos (principalmente, para justificar con ellos comportamientos discriminativos) y las de tipo sociocultural, según las cuales, los estereotipos tienen su origen en el sistema social y normativo; y, por tanto, llegan al individuo por medio de los procesos de socialización. En la primera corriente, el individuo adquiere un papel protagónico; en la segunda, más bien pasivo. Una tercera orientación, de carácter sociocognitivo, se sitúa entre ambas, al señalar que los estereotipos se forman y desarrollan por actividades cognitivas de los sujetos, si bien "la percepción de las diferencias entre grupos incluye, de una manera natural, diversos sesgos propios de los procesos de información sobre los demás" (Munné i Matamala, 1989: 324).

La difusión de unos datos en productos comunicativos puede ser considerada como un paso previo a la interiorización de dichos datos por alguno de los receptores efectivos (lectores, oyentes, televidentes). Desde otro ángulo, también podríamos concebir, tanto las descripciones que se publican y adquieren notoriedad, como las que ofrecemos en una conversación cotidiana, como el resultado de exteriorizar un conjunto de datos que antes hemos combinado mentalmente. Vista así, la narración publicada sería posterior y no previa a la interiorización de los datos. Por tanto, debe aceptarse, siguiendo al profesor Martin Serrano, que existen, al menos, estas dos formas de enfocar el análisis de los relatos, según se ponga

\footnotetext{
2 Entendemos por "productos comunicativos" aquellos que han sido fabricados para proveer de información pública a una comunidad.
} 
"el énfasis en el carácter generado o generador de la información” (Martín Serrano, 1986).

El carácter generado de los productos comunicativos se toma en cuenta cuando el análisis está orientado a encontrar en ellos las marcas que han dejado los responsables de su elaboración. Por contra, su condición de elementos generadores la tenemos presente cuando nos interesamos por saber si tales productos dejan alguna impronta en determinados miembros de una sociedad, al proporcionar unas visiones de la realidad que pueden ser asumidas como propias, en todo o en parte, por algunos receptores.

Tanto el primer enfoque como el segundo permiten arrojar alguna luz sobre fenómenos sociales como los mencionados anteriormente: la estereotipia, el prejuicio, etc.

El trabajo del que daremos cuenta a continuación sirvió como investigación para una tesis doctoral (Bernete, 1990) donde se abordó el análisis de unas narraciones en su condición de relatos generados en sociedades con diferentes dinámicas históricas, culturales, políticas, etc. Y, por tanto, explicando el sentido que cabe atribuir a los resultados teniendo en cuenta el lugar donde se han producido y usado tales narraciones.

El análisis ha consistido en probar si, efectivamente, es posible comprender algo mejor los endoestereotipos y exoestereotipos nacionales, relacionando su construcción comunicativa con motivaciones y necesidades que no están explícitas en los textos de historia, pero que pueden ser traídos a la luz, recurriendo a los métodos de análisis de contenido que aquí se han utilizado.

\section{EL OBJETO DE LA INVESTIGACION: LOS ESTEREOTIPOS NACIO- NALES OFRECIDOS EN LOS MANUALES DE HISTORIA}

Los productos comunicativos seleccionados para este análisis son libros de texto que se utilizan como material didáctico en cinco países distintos (Chile, Cuba, México, Perú y España), para explicar la asignatura de "Historia" en el nivel de enseñanza media. El relato de estos manuales se divide en lecciones referidas a las épocas que -según el criterio del autor o

Mediaciones Sociales, № 3, II semestre de 2008, pp. 73-90. ISSN electrónico: 1989-0494. 
autores del texto- se han sucedido en la vida de la comunidad cuya historia se difunde.

En la narración de lo acontecido en cada etapa histórica se ofrecen datos a propósito de personas, acontecimientos, leyes, problemas, etc. Para el objeto de este análisis, se recogieron las descripciones de los sujetos y las instituciones que aparecían mencionadas en el relato. A partir de ahora, a dichas unidades de análisis se les denomina personajes.

A los sujetos y organizaciones mencionados en la narración se les atribuye una función o papel social (por ejemplo: colonizador, gobernante, independentista), que el personaje desempeña -según el texto- dotado de unas determinadas características, personales y públicas: cualidades (ej. "honradez"), sentimientos (ej. "ira hacia los conquistadores”), derechos (ej. "posibilidad de ocupar cargos públicos”), etc.

\section{La construcción de estereotipos en la narración}

Los narradores estereotipan a los personajes que describen, cuando generan un efecto de homogeneización o de generalización:

- El relato lleva a cabo una homogenización (o desindividuación) cuando un personaje aparece descrito con los atributos que los autores de los textos también utilizan para caracterizar a otros personajes del mismo grupo.

- Por otra parte, induce una generalización cuando no especifica el grado en el que los personajes "poseen” los atributos. (Por ejemplo, debería afirmarse de unos sujetos que son "más/menos valientes que... (otros sujetos)" o "más/menos honrados que... (otros sujetos)". En lugar de atribuir características en términos relativos, suele asignarse un conjunto "a”, "b”... "x" de rasgos a los personajes identificados como miembros de los grupos "A", "B"... "X".

La homogeneización y la generalización son posibles criterios para objetivar el conjunto de atributos que componen el estereotipo de un colectivo (p.e., "los españoles”), luego, cabe establecer las semejanzas y diferencias entre las representaciones de los distintos colectivos analizados.

La investigación partió del supuesto siguiente: los narradores aluden a los personajes como miembros de una clase social, de una religión,

Mediaciones Sociales, № 3, II semestre de 2008, pp. 73-90. ISSN electrónico: 1989-0494. 
de una nacionalidad, de una etnia, etc. Según el grupo en el que sean incluidos, su descripción se formulará con unos u otros atributos.

Partiendo de este criterio, nuestro propósito ha sido poner de relieve las correspondencias existentes entre:

a) el conjunto de atributos empleados en la representación ${ }^{3}$ de cada personaje $\mathrm{y}$

b) la pertenencia del personaje descrito a un grupo determinado (por ejemplo, al colectivo de nacionalidad "A", o de raza "X").

En la investigación mencionada se ha estudiado la estructura de estereotipos "nacionales" y, por tanto, interesaba, en primer término, la correspondencia entre atributos y nacionalidades.

Ahora bien, las semejanzas y las diferencias entre las caracterizaciones de unos personajes y las de otros pueden estar relacionadas bien con su pertenencia a una u otra nación, bien con su inclusión en otra clase de agrupaciones (políticas, étnicas, etc.). La estructura de un estereotipo se pone de manifiesto cuando se descubren los filtros que han permitido la descripción de los personajes "A", "B" o "C", con los atributos "x", "y" o "z", en los libros de los países “1”, “2", “3”...”n”.

\section{Metodología EMPleAda: EL ANÁlisis de laS RELACiONES LÓGICAS}

Las técnicas de análisis que se utilicen en la investigación de las narraciones pueden ser utilizadas para estudiar las funciones mediadoras de las narraciones. Esto es, una "actividad que impone límites a lo que puede ser dicho y a las maneras de decirlo, por medio de un sistema de orden” (Martín Serrano, 1977: 54).

En tanto que las investigaciones no demuestren lo contrario, cabe aceptar que las limitaciones o constricciones formales en el código empleado en una práctica comunicativa institucional suelen ser congruentes con

\footnotetext{
3 Se entiende por "representación" un conjunto organizado de datos a propósito de alguna entidad: en nuestro caso, sujetos e instituciones mencionados en el relato.
}

Mediaciones Sociales, № 3, II semestre de 2008, pp. 73-90. ISSN electrónico: 1989-0494. 
las limitaciones o constricciones sociales: por ejemplo, se ha podido llegar a mostrar recurriendo al análisis de las narraciones que unos prejuicios compartidos en el seno de una comunidad se reflejan en las relaciones lógicas (inclusión, implicación, exclusión, etc.) que mantienen entre sí los datos de referencia contenidos en las narraciones.

El análisis de las relaciones lógicas es un instrumento con el cual puede objetivarse la estructura de los modelos comunicativos, donde se expresan cada una de las visiones del mundo propuestas por los mediadores culturales. Ello es posible con este método porque son códigos lógicos los que se emplean en la comunicación para ejercer el control cognoscitivo. Otras instituciones, no especializadas en la producción de comunicación, ejercen el control sobre el conocimiento sirviéndose de códigos sociales (autorizaciones, prohibiciones, etc.).

Para investigar la formación de los estereotipos en la televisión se examinaron las relaciones lógicas que cada uno de los atributos mantenía con todos los demás. Con este fin, se distinguieron cuatro clases de funciones: Determinación, interdependencia, constelación y exclusión ${ }^{4}$.

\footnotetext{
${ }^{4}$ Las tres primeras fueron utilizadas anteriormente por Hjelmslev (1968). Martín Serrano incorporó al análisis la "exclusión", cuando la presencia de un elemento supone necesariamente la no presencia de otro elemento. En L'ordre du monde à través la Tv (1976) se investiga por primera vez el sistema de formación de estereotipos en la televisión, con las mismas funciones lógicas que se han utilizado en esta investigación para estudiar la formación de estereotipos en los libros de texto de Historia. El método de análisis lógico es "el único que puede recoger la complejidad de lo social como una totalidad”. Según Martín Serrano, en los análisis lógicos pierde su sentido (metodológico) la diferencia entre "análisis cualitativos” y "análisis cuantitativos".
} 


\begin{tabular}{|c|c|}
\hline \multicolumn{2}{|l|}{$\begin{array}{l}\text { Sean los datos: } \\
\text { A = "nacionalidad española" } \\
\text { B = "religiosidad" }\end{array}$} \\
\hline $\begin{array}{l}\text { La determinación se re- } \\
\text { presenta de las dos formas } \\
\text { siguientes: }\end{array}$ & \\
\hline $\mathrm{A} \longrightarrow \mathrm{B}$ & $\begin{array}{l}\text { Los españoles siempre aparecen descritos como } \\
\text { religiosos, pero no todos los personajes que apare- } \\
\text { cen como religiosos son españoles. }\end{array}$ \\
\hline $\mathrm{A} \leftarrow \mathrm{B}$ & $\begin{array}{l}\text { La religiosidad se atribuye sólo a los españoles, pero } \\
\text { no es la única característica atribuida a los persona- } \\
\text { jes de nacionalidad española. }\end{array}$ \\
\hline $\begin{array}{l}\text { La interdependencia se } \\
\text { representa del siguiente } \\
\text { modo: } \\
\mathrm{A} \quad \longleftrightarrow \text { B }\end{array}$ & $\begin{array}{l}\text { Todos los españoles están descritos como religiosos } \\
\text { y todos los personajes a los que se atribuye religio- } \\
\text { sidad son españoles. }\end{array}$ \\
\hline $\begin{array}{l}\text { La constelación se indica } \\
\text { de la manera siguiente: } \\
\mathrm{A} \text { 프 B }\end{array}$ & $\begin{array}{l}\text { A los españoles se les atribuye "religiosidad" con } \\
\text { una frecuencia significativa (pero no siempre). A su } \\
\text { vez, la asignación de "religiosidad" recae frecuente- } \\
\text { mente (pero no siempre) sobre personajes españoles. }\end{array}$ \\
\hline $\begin{array}{l}\text { La exclusión se refleja } \\
\text { como sigue: } \\
\mathrm{A} \sqsupset \sqsubset \mathrm{B}\end{array}$ & $\begin{array}{l}\text { Los españoles nunca son descritos como personajes } \\
\text { religiosos. O bien, dicho de otro modo, la atribución } \\
\text { de "religiosidad" jamás recae sobre personajes de } \\
\text { nacionalidad española. }\end{array}$ \\
\hline
\end{tabular}

\section{VERIFICACIONES MÁS RELEVANTES DEL CONJUNTO DEL ANÁLISIS}

\subsection{La identificación y legitimación de un colectivo nacional}

El libro de historia proporciona un relato de lo que acontece y de lo que aconteció, que supone necesariamente una representación. El conocimiento de dicha visión del acontecer sirve para desvelar cómo los narradores median entre los sucesos que pasan y los valores que traen a colación para juzgar tales sucesos. Toda vez que el relato de historia cumple una función educativa, resulta ser un material adecuado para comprobar de 
qué modo llevan a cabo su labor mediadora las instancias gubernamentales que aprueban dichos textos; las cuales se incluyen -con los Medios de Comunicación de Masas, la Iglesia, etc.- entre las instituciones que tienen a su cargo la producción y difusión de comunicación pública.

Nuestra tarea ha consistido en descomponer un discurso mediador y reconstruirlo con otro orden que permite desvelar un sentido no explícito en el propio relato. Por ejemplo: en los libros de historia no se afirma expresamente que los actores chilenos que gobiernan después de la independencia tengan características similares a las que poseen los españoles que lo hacen antes de la independencia. Sin embargo, como en los manuales se describe a los gobernantes de ambas nacionalidades de forma semejante (preservando alguna diferencia) podemos afirmar que aparecen como miembros de un colectivo que abarcaría a los primeros y a los segundos.

Los autores de los textos de historia, cuando seleccionan unos aconteceres, unos datos relativos a dichos aconteceres y unas formas determinadas de organizar los datos, ofrecen en los productos comunicativos resultantes, unas cosmovisiones. Estas visiones del mundo, representan las interpretaciones de la realidad que las autoridades educativas, aunque sea de manera inconsciente, tienen por apropiadas.

En todos los países, los manuales de historia legitiman acciones, personas, instituciones, relaciones de autoridad, valores, creencias, etc. Al hacerlo, se proporcionan a la comunidad unos modelos de los respectivos endogrupos y sus correspondientes exogrupos. Pero subsiste la cuestión de identificar cuál es el grupo legitimado en cada caso, pues en toda sociedad existen intereses contrapuestos entre unos colectivos y otros; razón por la cual es necesario investigar si los autores de los textos narran la historia adoptando la perspectiva de un grupo determinado y quiénes lo integrarían según el relato.

La legitimación de unos actores -individuales y colectivos-, frente a otros, puede atribuirse a que los libros de texto están orientados a ofrecer determinadas versiones del acontecer que respeten las normas y valores en cuya preservación se interesan las instituciones del sistema educativo. Los mediadores han de construir el relato manejando las tensiones que existan entre: a) los acontecimientos ocurridos hasta la etapa en que se escribe y b) la organización social con la que cuenta la comunidad en dicha 
etapa. Estas tensiones son, histórica y coyunturalmente, de distinta naturaleza en cada país. Por este motivo, también son diversas las posiciones adoptadas por los autores de los textos utilizados en el año académico 1985/86:

A) Los mediadores que intervienen en la narración de "La Historia de Chi$l e$ " adoptan el punto de vista de una "comunidad" -"real o artificial", en palabras de Hobsbawn (1988)- que podríamos llamar "hispano-chilena". Se legitiman las actuaciones de quienes ejercen la función de gobernar (antes y después de la independencia), excepto las de los "gobernantes revolucionarios". (La descalificación de los "gobernantes revolucionarios", obviamente, tiene que ver con la justificación del golpe de Estado del General Pinochet, que, como es sabido, permanece en el poder en 1985/86, cuando se recoge el material de análisis). Los enemigos -antihéroes en el relatoson: por un lado, los gobiernos no conservadores y, por otro lado, los dirigentes de los países limítrofes, con los que Chile mantuvo enfrentamientos políticos y militares.

B) El relato de "La Historia de Cuba" está orientado a conseguir la aceptación del orden social establecido con la Revolución socialista, que, a su vez, se presenta como la culminación del proceso de independencia iniciado en el S. XIX. Los protagonistas-héroes son quienes han luchado por la Independencia, primero, y por la Revolución, después. Con independentistas y revolucionarios se crea un único colectivo, que aparece como prototipo de la nación cubana. Los antagonistas-antihéroes son, en esta narración, los mencionados como "conquistadores", "colonizadores" o "antirevolucionarios". Todos ellos están descritos como si formasen otro colectivo, el de anticubanos, que, obviamente incluye personajes nacionales.

C) En los manuales de "Historia del Perü" confluyen distintas concepciones acerca de qué es y cuándo comienza a existir "lo peruano". Dicha confluencia, como es lógico, dificulta la diferenciación del grupo nacional peruano, respecto a otros colectivos. Para lograr esta diferenciación del endogrupo, no se utiliza el recurso empleado en los libros de Chile y Cuba, que consiste en adjudicar el protagonismo histórico a un subgrupo nacional, definido por su posición política. La sociedad peruana se presenta, en los libros, cohesionada, en cuanto que no existen referencias a conflictos intragrupales, de carácter social. En el Perú contemporáneo, los antagonismos son partidarios (entre liberales y conservadores) o militares (con Chile), pero nunca entre clases sociales.

Mediaciones Sociales, № 3, II semestre de 2008, pp. 73-90. ISSN electrónico: 1989-0494. 
D) La narración de la "Historia de México" pone de manifiesto la existencia de divisiones sociales y de conflictos endogrupales. Los enfrentamientos entre mexicanos ocupan la mayor parte del relato, a diferencia de lo observado en los textos de Perú. Los conflictos entre políticos mexicanos de tendencia distinta dejan de mencionarse en la narración cuando el relato da cuenta de la fundación del P.R.M. (antecesor del actual P.R.I.). Este partido no aparece descrito explícitamente como instaurador del consenso o la cohesión endogrupal. Sin embargo, esa es la interpretación que sugiere el relato, dejando de aludir a conflictos endogrupales cuando se convierten en objetos de referencia los acontecimientos transcurridos a partir de 1940. La mediación, en los textos de México analizados, sitúa en dos planos diferentes a los "protagonistas" de la vida mexicana:

- En un plano, a los políticos del pasado (hasta 1940), como personas que encabezaban grupos y llevaban a cabo enfrentamientos con otros actores.

- En otro plano, a los gobiernos del presente (desde 1940 hasta 1983), como administraciones que llevan a cabo actividades institucionales.

E) Por último, los libros españoles utilizados en 1985 (y aprobados en 1976), cuando narran la "Historia de los países hispánicos" están orientados a consolidar una visión de los acontecimientos históricos (Conquista, Colonización, Independencia, etc.) que no cuestione el comportamiento de las personas y, sobre todo, de las instituciones españolas en el continente americano. El relato no se ocupa de definir la nacionalidad española, frente a otras, ni cuenta la "Historia de España". Incide en que la conquista de América produjo un cambio en el rumbo histórico de las tierras descubiertas y en que la posterior independencia política de los países hispánicos ha significado, para estos, el sufrimiento de un nuevo colonialismo económico y cultural ejercido por otras metrópolis.

\subsection{Matices que deben incorporarse a la tesis del favoritismo endo- grupal.}

Tanto las narraciones de los libros de texto como las que ofrecen a los niños sus padres, amigos, profesores, medios de comunicación de masas, etc. pueden girar en torno a la separación entre "lo del grupo propio" y "lo de grupos ajenos". El etnocentrismo se manifiesta, entre otras formas, como la tendencia a tener una representación más positiva del grupo pro-

Mediaciones Sociales, № 3, II semestre de 2008, pp. 73-90. ISSN electrónico: 1989-0494. 
pio (clase social, nacionalidad, profesión, etc.) que de los grupos ajenos. Esa representación etnocéntrica se usa para "justificar" los sentimientos de superioridad que cada grupo alimenta en sus comparaciones con otros colectivos.

Algunos autores ${ }^{5}$ han señalado que los fenómenos "etnocentrismo" y "estereotipia" no van necesariamente unidos. Pero los resultados de la mayor parte de las investigaciones confirman que "los sujetos discriminan a favor del endogrupo y en contra del exogrupo y muestran actitudes más positivas hacia el primero que hacia el segundo" (Morales Domínguez, 1989: 59). La repetida confirmación de tal hipótesis ha dado lugar al consenso en torno al "sesgo endogrupal" o "favoritismo endogrupal" 6 .

La investigación realizada ha confirmado la existencia de estereotipos favorables del endogrupo en los libros de Chile, Cuba, Perú y España, pero no así en los libros de México, donde las referencias a los enfrentamientos políticos y militares entre los propios mexicanos y las cualificaciones de "incapacidad política" de los cargos públicos, configuran un estereotipo desfavorable del grupo propio.

Debe tenerse en cuenta que en "La Historia de México" la representación negativa se ofrece a propósito de los políticos y otras personas que intervenían en la vida pública mexicana hasta la fundación del P.R.M., pero no se ofrece a propósito de los políticos y otros personajes públicos contemporáneos, de los cuales no hay descripciones.

El análisis de los textos de los cinco países seleccionados nos obliga a incorporar los siguientes matices, a propósito del favoritismo endogrupal:

\footnotetext{
5 Karlins, Coffman y Walter insisten en esta idea, al afirmar que "el etnocentrismo, evidentemente, no es inherente a la estereotipia, en sí misma” (1969: 12, citado en Sangrador, 1981: 99).

6 "El apoyo experimental con que cuenta la tendencia al favoritismo endogrupal se refiere a diferentes aspectos de la misma. Se han encontrado las características físicas más deseables (Doise et al., 1972), así como las creencias más parecidas a las suyas (Allen, Wilder, 1975). Además, se tiende a evaluar más positivamente las tareas realizadas por el endogrupo (Hinkle y Shopler, 1979), a asignar items más positivos a sus miembros (Howard y Rothbart, 1978) y a otorgarles los valores estimados más deseables (Locksley et al., 1980), todo ello en comparación con el exogrupo" (Munné i Matamala, 1989: 345).
}

Mediaciones Sociales, № 3, II semestre de 2008, pp. 73-90. ISSN electrónico: 1989-0494. 
a) La nacionalidad no es un criterio que asegure en todos los libros de historia una caracterización favorable del endogrupo.

b) En los casos en que se han observado los sesgos, éstos no se producen siempre en favor de todo el grupo nacional. En los manuales de determinados países (como Chile, Cuba y España) se opera también con otros criterios distintos a la nacionalidad.

c) Cuando se propone una representación crítica o desfavorable del endogrupo, dicha representación tampoco está referida a todo el conjunto nacional, sino a un colectivo determinado que adquiere mayor protagonismo en el relato de la historia. Así ocurre en la narración de los libros mexicanos con quienes son descritos como políticos, ya sean gobernantes o no.

\subsection{El recurso a diversos criterios para representar a los colectivos de personajes}

En los libros de texto de Historia que se han analizado, la NACIONALIDAD no es el único criterio seguido para conformar la representación de cada grupo de personajes. Por el contrario, sólo una parte de las características asignadas a los actores es explicable por la pertenencia de éstos a una comunidad nacional; otra parte puede ser explicada como consecuencia de haber descrito a los personajes en tanto que miembros de agrupaciones diferentes. Por ejemplo, un movimiento político o una "patria cultural”.

En un mismo relato, se recurre a distintos criterios para representar a los personajes nacionales, dependiendo del uso descriptivo ${ }^{7}$ que se haga de los atributos. Por ejemplo, en los manuales de Cuba, se observa lo siguiente:

- Por una parte, los atributos se utilizan para establecer similitudes entre personajes "cubanos" e "iberoamericanos" no cubanos, que comparten con los primeros alguna característica distinta de la nacionalidad, como la pertenencia al "Movimiento obrero y revolucionario". Cuando se da este uso a

\footnotetext{
Por "usos descriptivos" debe entenderse, en este contexto, las diferentes utilidades que pueden tener los atributos empleados en la descripción de los personajes. Por ejemplo: los atributos se usan para estereotipar al colectivo de personajes que pertenecen al mismo país que los libros; para estereotipar a los personajes de otros países; para establecer semejanzas o/y diferencias entre las representaciones de los distintos grupos, etc.
}

Mediaciones Sociales, № 3, II semestre de 2008, pp. 73-90. ISSN electrónico: 1989-0494. 
los atributos, se está recurriendo al criterio del ROL, el cual permite presentar al grupo prototipo de la nación como "integrado" en el contexto internacional.

- Por otra parte, los atributos también se utilizan para establecer diferencias entre "cubanos" y todos los "no cubanos". Cuando se da este uso a los atributos, se está recurriendo, obviamente, al criterio de la NACIONALIDAD, aunque los "no cubanos" sean personajes iberoamericanos que comparten con los cubanos algunas otras características (como la pertenencia al "Movimiento obrero y revolucionario").

El recurso a más de un criterio, en el conjunto del relato, indica que la construcción comunicativa de la identidad en los manuales de historia, se lleva a cabo representando a los personajes como pertenecientes a colectivos de distinta naturaleza, pero compatibles entre sí, y no sólo como miembros del grupo nacional.

Los análisis que se diseñen para estudiar la formación de representaciones sociales a propósito de grupos humanos, deben prever la existencia de diversos criterios diferenciales para poder explicar las constantes y las variantes observadas en la construcción de tales representaciones.

\subsection{La importancia de tomar en cuenta el "rol" que desempeñan los actores}

La función social o ROL de los personajes tiene singular importancia para explicar el modo con el cual se estereotipa en los textos de historia, por un doble motivo:

a) En los manuales de cada país puede aparecer: un número " $\mathrm{x}$ " de personajes que desempeñan el rol "A"; un número "y" de personajes que desempeñan el rol "B" y así sucesivamente. El protagonismo histórico suele estar relativamente concentrado en los actores que ejercen unas funciones sociales concretas, en detrimento del protagonismo de aquellos otros actores que desempeñan papeles distintos. Por ejemplo, en una narración se mencionan más "colonizadores" que "independentistas" y en otras sucede a la inversa.

b) A los personajes se les caracteriza con rasgos que le son atribuidos en el desempeño de un rol determinado. El rol puede actuar como

Mediaciones Sociales, № 3, II semestre de 2008, pp. 73-90. ISSN electrónico: 1989-0494. 
elemento del cual depende la asignación de cualidades, sentimientos, derechos, obligaciones, etc. Por ejemplo: quienes desempeñan el papel de "independentistas" son descritos como "patriotas" (entre otros rasgos), quienes ejercen de "gobernantes" aparecen como "corruptos", etc.

\subsection{Las delimitaciones de la "identidad"}

La población de los países latinoamericanos puede ser caracterizada de muy diversas formas, puesto que son muchos los criterios diferenciales (etnia, religión, lugar de procedencia, etc.) que podrían tomarse en cuenta. La existencia de múltiples combinaciones de criterios debe ser considerada para valorar la opción que se ha elegido: en los relatos analizados, los mediadores han optado por describir a los personajes según la nacionalidad y el rol, entre las alternativas posibles. Esta elección merece, por nuestra parte, las reflexiones siguientes:

A) Elegir el criterio "NACIONALIDAD" supone privilegiar una manera de constituir colectivos humanos (en nación), frente a la posibilidad de preservar entidades de naturaleza distinta: comunidades culturales, religiosas, económicas, raciales o políticas, de menor o mayor extensión que el Estado nacional. Por ejemplo, la constitución de una "comunidad iberoamericana”. Los libros analizados están lejos de promulgar una entidad de este carácter.

En el diseño de esta investigación se previó la recogida de un rasgo que apenas aparece en los manuales, por lo cual no pudo ser explotado: se trata de la ETNIA de los personajes. Al describir a los actores como miembros de una nación, se ha silenciado su pertenencia a grupos étnicos. Dicho de otro modo: la "identidad nacional" se está construyendo comunicativamente sobre un ocultamiento de identidades étnicas (entre otras), cuya existencia real no reflejan los libros de texto. Puede afirmarse que, al menos, los manuales de Historia estudiados no contribuyen a un conocimiento etnológico del área geográfica donde se utilizan.

B) Elegir el criterio "ROL" supone, en la práctica de la elaboración del relato, reducir la colectividad nacional a la colectividad políticomilitar que gobierna o lucha por el gobierno. La historia del país suele estar contada como una sucesión de gobernantes y opositores o como una historia bélica, en el contexto de las naciones limítrofes y de las colonizadoras.

Mediaciones Sociales, № 3, II semestre de 2008, pp. 73-90. ISSN electrónico: 1989-0494. 
La nueva reducción es complementaria de la anterior, puesto que concretar la "identidad" en "identidad nacional" exige proponer a determinados personajes como prototipos de la nación. Estos actores no se seleccionan por su naturaleza de indios, mulatos, mestizos, hombres, mujeres, pintores, músicos, etc., sino dependiendo del papel social que han desempeñado (o, al menos, se les atribuye) en la constitución del Estado nacional.

Los manuales de historia no ponen de manifiesto la diversidad cultural, étnica, axiológica, etc. de los grupos humanos que conviven en el interior de los Estados chileno, cubano, mexicano, peruano y español. Obviamente, pensamos que la diversidad debe reflejarse, en la medida en que lo permitan los límites de los productos comunicativos, sin que las diferentes peculiaridades de cada grupo se describan de tal modo que puedan generar o justificar actitudes prejuiciosas o comportamientos discriminatorios.

Nuestro estudio sigue un procedimiento propio de la investigación objetivada. Valida conclusiones que, en algunos casos, fueron intuidas con anterioridad. Varios escritores de genio tuvieron la lucidez de acertar en el análisis de la identidad nacional de sus respectivos países sin otro bagaje que su capacidad de observación y reflexión. La convergencia entre los resultados de esta investigación de contenido y las tesis de los intelectuales latinoamericanos hace sospechar que las representaciones colectivas están difundidas en muchos ámbitos (culturales, educativos, comunicativos) y que, probablemente, también aparecerían contenidos semejantes si se analizaran productos distintos de los libros de historia.

Los textos de Historia que los adolescentes estudian en el bachillerato intervienen en la visión del mundo junto con otros productos (películas, discos, tebeos, etc.) y agentes (profesores, padres, amigos, etc.). Cada una de esas instancias enculturizadoras puede transmitir representaciones, sean o no prejuiciosas, sobre determinadas razas, clases sociales, etc. y, por supuesto, sobre países o áreas geográficas. La posible influencia de los libros de historia se inscribe en el juego de todas esas fuentes alternativas de representaciones. Si el resultado fuese la interiorización, por parte de los sujetos, de actitudes discriminatorias, en favor o en contra de alguno de los grupos representados, se produciría la paradoja de que la comunicación se constituyese en inductora de la incomunicación entre los hombres. 
De cualquier modo, los juicios de valor cargados de xenofobia o de desprecio no suelen aparecer explícitos en los textos, lo cual no es óbice para que esa valoración esté implícita en la estructura narrativa y sea igualmente eficaz como estímulo prejuicioso. Por este motivo, entendemos que la objetivación de los estereotipos que se transmiten a través de todas las vías de socialización de los individuos es un paso necesario para evitar prejuicios y xenofobias.

\section{BIBLIOGRAFÍA}

BERNETE, Francisco (1990): Los estereotipos de la comunidad iberoamericana. Madrid: Universidad Complutense de Madrid. Tesis Doctoral.

HJELMSLEV (1968): Prolégomènes à une theorie du langage. Paris: Minuit.

HOBSBAWN, Eric J. (1988): L'invent de la tradició. Barcelona: Eumo.

MARTÍN SERRANO, Manuel (1976): L'ordre du monde à través la Tv. Strasbourg, Lilles: Univ. L. Pasteur, Presses Universitaires.

MARTÍN SERRANO, Manuel (1977): La mediación social. Madrid: Akal.

MARTÍN SERRANO, Manuel (1986): La producción social de comunicación. Madrid: Alianza ( $\left.1^{\mathrm{a}} \mathrm{ed}\right)$.

MORALES DOMÍNGUEZ, J.F. (1989): "Identidad social y personal", en RODRÍGUEZ, A. y SEOANE, J.: Creencias, Actitudes y Valores. V. 7 Tratado de Psicología General de J. MAYOR y J. PINILLOS. Madrid: Alhambra.

MUNNÉ i MATAMALA, Frederic (1989): "Prejuicios, estereotipos y grupos sociales”, en RODRÍGUEZ, A. y SEOANE, J.: Creencias, Actitudes y Valores. V. 7 Tratado de Psicología General de J. MAYOR y J. PINILLOS. Madrid: Alhambra, pp. 315-363.

RODRÍGUEZ A. y SEOANE J. (1989): Creencias, Actitudes y Valores. V. 7 Tratado de Psicología General de J. MAYOR y J. PINILLOS. Madrid: Alhambra.

SANGRADOR, J.L. (1981): Estereotipos de las nacionalidades y regiones de España. Madrid: CIS.

Mediaciones Sociales, № 3, II semestre de 2008, pp. 73-90. ISSN electrónico: 1989-0494. 


\section{PARA CITAR ESTE TRABAJO EN BIBLIOGRAFÍAS:}

BERNETE GARCÍA, Francisco (2008): "El estudio de los estereotipos a través del análisis de relatos”, Mediaciones Sociales. Revista de Ciencias Sociales y de la Comunicación, $\mathrm{n}^{\circ}$ 3, segundo semestre de 2008, pp. 73-90. ISSN electrónico: 19890494. Universidad Complutense de Madrid.

Disponible en: http://www.ucm.es/info/mediars

\section{${ }^{(*)}$ El autor}

Francisco Bernete García se doctoró en la UCM con una Tesis sobre Los estereotipos de la comunidad iberoamericana (1990). Es Profesor Titular de Teoría y Sociología de la Comunicación en la Universidad Complutense de Madrid. En posgrado, imparte docencia relacionada con los efectos de la comunicación, el análisis de contenido y la comunicación pública en la sociedad multiétnica.

Ha participado en varias investigaciones sobre la juventud en España desde 1990. Entre ellas, Los valores actuales de la juventud española (1991) e Historia de los cambios de mentalidades de los jóvenes 1960-1990 (editado en 1994). Es coautor del libro Jóvenes, relaciones familiares y tecnologías de la información y la comunicación (2004) y director del estudio Comunicación y lenguajes juveniles a través de las TIC (2006). Recientemente ha coordinado el núm. 78 de la Revista de Estudios de Juventud, monográfico dedicado a "Culturas y lenguajes juveniles".

Ha sido Director de la Sección Departamental de SOCIOLOGÍA IV y actualmente es responsable del Máster en Comunicación Social de la UCM. 